\title{
An Intelligent Biogas Digester Monitoring System Based on Wireless Sensor Network Hao Chen ${ }^{1, a}$, Qianyun Chu ${ }^{2, b}$
}

School of Electronics and Information Engineering, Anhui University, Hefei, 230601, China

aemail: chenhao@ahu.edu.cn, bemail: chuqianyun1995@163.com

Key Words: Wireless Sensor Network, ZigBee, GPRS Intelligent Gateway, Cloud Server

\begin{abstract}
Biogas is deemed as a kind of superior and renewable resource. It is urgent for Supervisory Departments to adopt a real-time monitoring system which may process data promptly so as to reduce the potential danger and improve the level of automation. In this paper, an intelligent biogas digester monitoring system based on wireless sensor network is designed. Sensor nodes collect the environmental information and transmit to the coordinator nodes by ad hoc, the environmental information is uploaded to cloud server through the GPRS intelligent gateway after being processed, so the users may acquire the information of biogas digesters in time. After using and testing, the system provides long-term and reliable service, and it has superior anti-interference ability, by means of wireless sensor networks, the system accomplishes intelligent and informational management of biogas digesters.
\end{abstract}

\section{Introduction}

Biogas is regarded as a kind of superior and renewable resource, using biogas is benefit for economic and ecological development [1]. However, traditional biogas digesters are lack of automation and safety performance, when biogas digesters are out of order, problems are difficult to submit to maintenance departments. Therefore, the specific analysis of conditions and regular patterns [2], timely collection of environmental information and reasonable estimation is of great significance for the extension of biogas digesters.

Wireless sensor networks have attracted significant attention among researchers and practitioners, and it have been shown to have a high potential to be the hotspot technology in environmental monitoring area [3]. A wireless sensor network refers to a collection of sensor nodes with the capability of wireless communication. The variable numbers of static or dynamic sensors form a transmission network by means of self-organization and multi-jump [4]. Wireless sensor network is able to collect and monitor environmental information such as temperature, humidity, pressure and so on, the information is transmitted by wireless network after being processed in order to realize the fusion of data.

The applications of wireless sensor network generally involve deploying a large number of sensor nodes in specific space, examples include precision agriculture monitoring [5], patient monitoring [6], environmental monitoring [7], and coal mine monitoring system [8]. However, these strategies may not satisfy long-term and reliable service. In this paper, a kind of intelligent biogas digester monitoring system based on wireless sensor network was designed. Data acquired precisely by wireless sensor networks is transmitted to control center and cloud server through GPRS intelligent gateway, so as to build a data collecting and early warning system [9]. Users can access the database and accomplish remote control easily by mobile device.

In the follow, we will introduce the architecture of this system in section II, then recommend hardware design and software design in section III and IV. Respectively, in section V, simulation and experimental results of this system are given and conclusion is in section VI.

\section{Architecture of Monitoring System}

An intelligent biogas digester monitoring system based on wireless sensor network is mainly made up with three parts: distributed wireless sensor networks, GPRS intelligent gateway, cloud 
server as shown as Fig. 1. Data collected by Distributed sensor networks is transmitted to GPRS intelligent gateway so as to finish characteristic level data fusion, the information can be displayed on mobile devices.

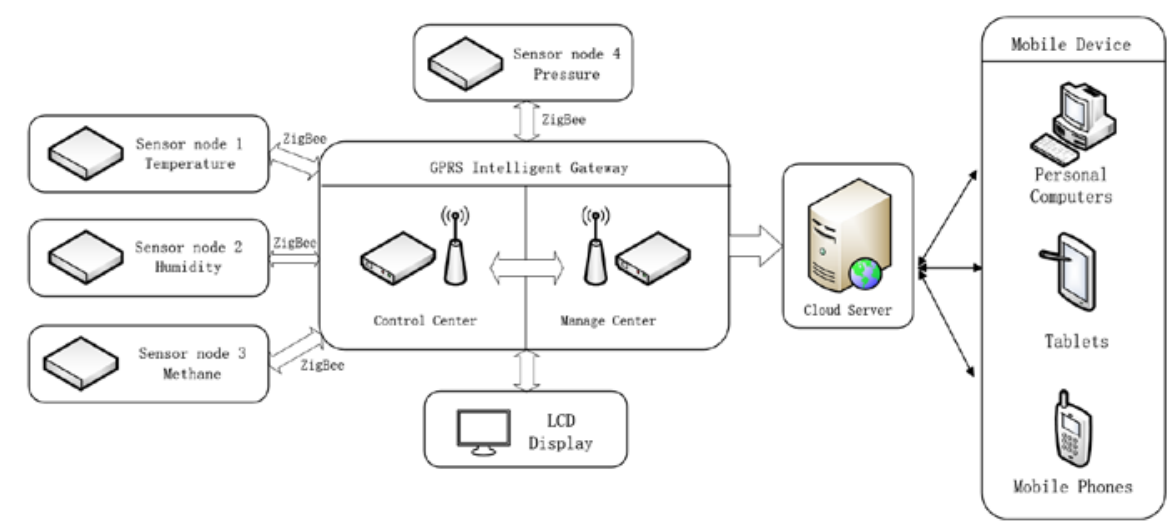

Fig. 1. Architecture of monitoring system

1. Distributed wireless sensor networks

Distributed wireless sensor networks consists of sensor nodes and ZigBee module. Sensor nodes collect environmental information by means of self-organization and multi-jump. Data is transmitted to control center and manage center by cooperation of ZigBee cluster nodes. Wireless sensor networks of system collects environmental data when users request, it also can collect and transmit information automatically as period setting. When a sensor node is set as a routing node, it may transfer the data packet, so as to expand the coverage of sensor networks and the capacity of data transmission.

2. GPRS intelligent gateway

GPRS intelligent gateway is responsible for bidirectional interaction and effective cooperation between wireless sensor networks and cloud server [10]. GPRS intelligent gateway mainly consists of control center and manage center. Control center forms ZigBee network, transmit information collected by sensor nodes to manage center and sends instructions to sensor nodes. Manage center displays real-time information collected by control center and stores the data to SD card.

3. Cloud server

In this paper, data is transmitted from GPRS intelligent gateway to cloud server, so as to finish deeper hierarchical data fusion, the cloud server will trigger the alarm automatically when potential danger is found. The Supervisory Departments will take more effective measures by using the monitoring system.

\section{Hardware Design of System}

1. Hardware design of sensor nodes

Sensor nodes mainly consist of data collection module, data process module, data transmission module and power management module shown as Fig. 2. The hardware of routing nodes is same as sensor nodes. This paper chooses CC2530 SOC produced by TI as the core chip of sensor nodes [11], CC2530 SOC has superior receiving sensitivity and anti-interference, the sensor nodes based on CC2530 contribute to real-time communication and low power consumption. 


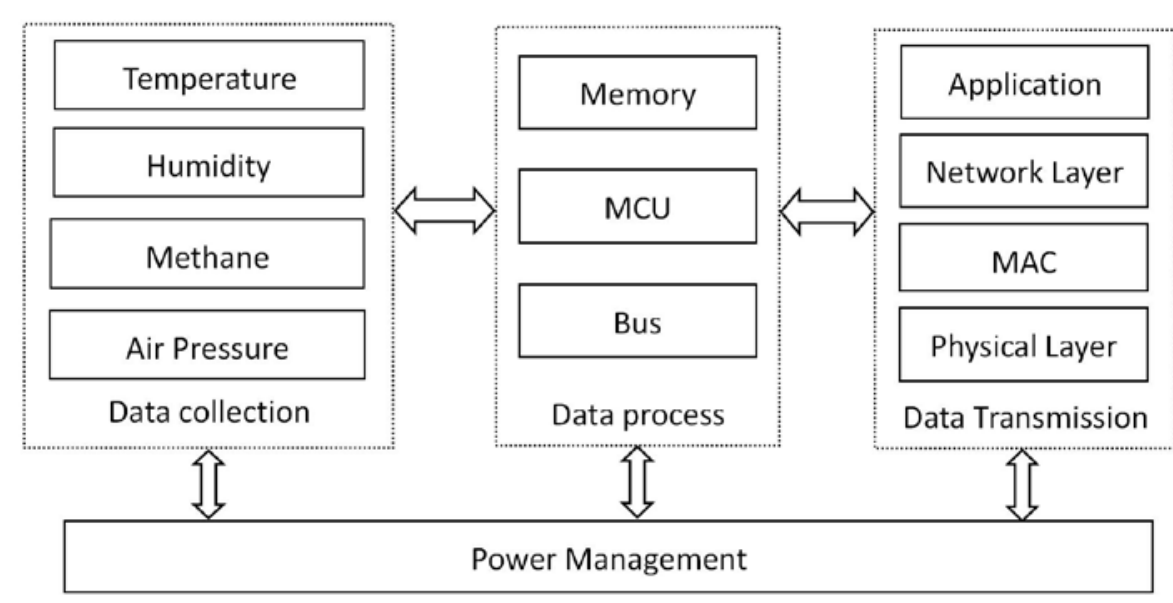

Fig. 2. Structure of sensor nodes

2. Hardware design of GPRS intelligent gateway

The GPRS intelligent gateway contains control center and manage center shown as Fig. 3. Control center is responsible for the establishment and adjustment of ZigBee networks. Control center interacts with manage center by receiving sensor data and sending instructions to nodes. Users are able to change settings of ZigBee network, GSM module and LCD module at same time through manage center. Data is able to be saved to SD card as users' requirements so as to realize the convenient management.

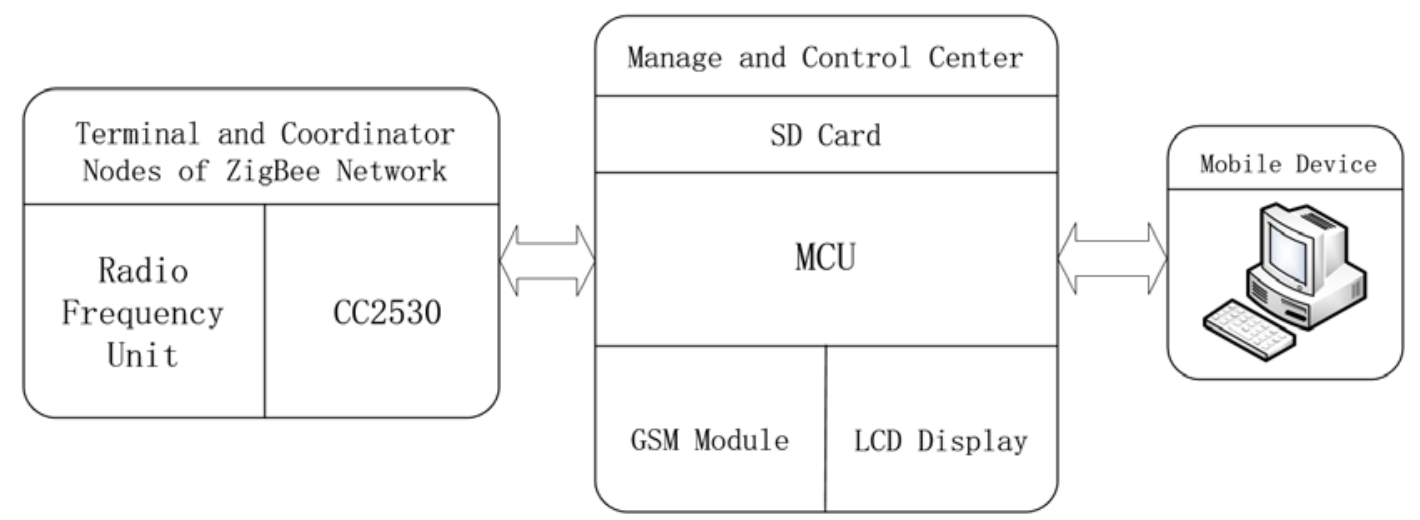

Fig. 3. Structure of GPRS intelligent gateway

\section{Software Design of System}

The software design of system contains three parts: sensor nodes, intelligent gateway and manage center. This paper mainly talks about the flowcharts and the algorithms of the program.

1. Software Design of Sensor Nodes

The signal communication between sensor nodes and control center is based on master-slave pattern, control center sends requirements upon fixed periods proactively, and the sensor nodes transmit data to gateway by means of interruption. Besides, the settings of transmitting period can be changed at control center. The flowchart of information transmitting is shown as Fig. 4. 


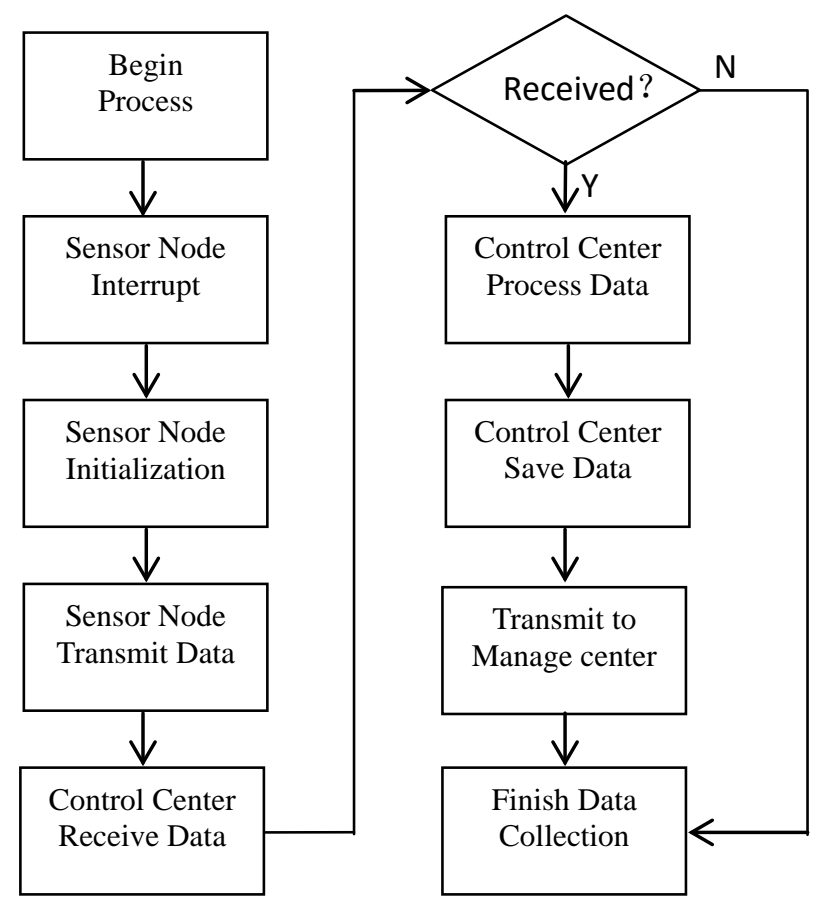

Fig. 4. The flowchart of information transmitting

2. Algorithms of Intelligent Gateway

The software design of intelligent gateway is mainly about the algorithms to figure out the fusion estimates of control center [12]. The data fusion of multi-sensor nodes can be regarded as a multivariable objective function with constraints. The objective function can be written as follows:

$$
Y\left(\alpha_{i}\right)=y\left(\alpha_{i}\right)+\lambda \sigma\left(\alpha_{i}\right)
$$

The constraints can be written:

$$
\sigma\left(\alpha_{i}\right)=\sum_{i=1}^{n} \alpha_{i}-1
$$

Calculate partial derivative equations with respect to $\alpha_{i}$ :

$$
\frac{\partial Y}{\partial \alpha_{i}}=\frac{d y}{\alpha_{i}}+\lambda=2 \alpha_{i} \sigma_{i}^{2}+\lambda(i=1,2, \cdots, n)
$$

If $\frac{\partial Y}{\partial \alpha_{i}}=0$,so $Y$ gets minimum value:

$$
\begin{aligned}
& \alpha_{i}=\frac{\lambda}{2 \sigma_{i}^{2}}(i=1,2, \cdots, n) \\
& \lambda=\frac{2}{\sum_{i=1}^{\mathrm{n}} \frac{1}{\sigma_{i}}}(i=1,2, \cdots, n)
\end{aligned}
$$

From Equations (4), (5) ,we can obtain:

$$
\alpha_{i}=\frac{\lambda}{\sigma_{i}^{2} \sum_{i=1}^{n} \frac{1}{\sigma_{i}^{2}}}(i=1,2, \cdots, n)
$$

The twice-weighed average can be concluded by Equations above:

$$
x_{i}=\sum_{i=1}^{n} \alpha_{i} w_{k}^{i} v_{j}^{i}
$$

Where $x_{i}$ is the fusion estimate at moment $i ; \alpha_{i}$ is the fusion weights. $w_{k}^{i}$ Is the best proportional weight of sensor $i$ at moment $k ; v_{j}^{i}$ are the sum of the observations and the 
estimates of sensor $i$ at moment $j$.

3. Software design of manager center

The manager center of system is responsible for five aspects: display the information of environment with LCD; transmit data to cloud server; save the data to SD card; finish the settings of ZigBee network; receive the instruction and send it to control center. The flowchart of manage center is shown as Fig.5.

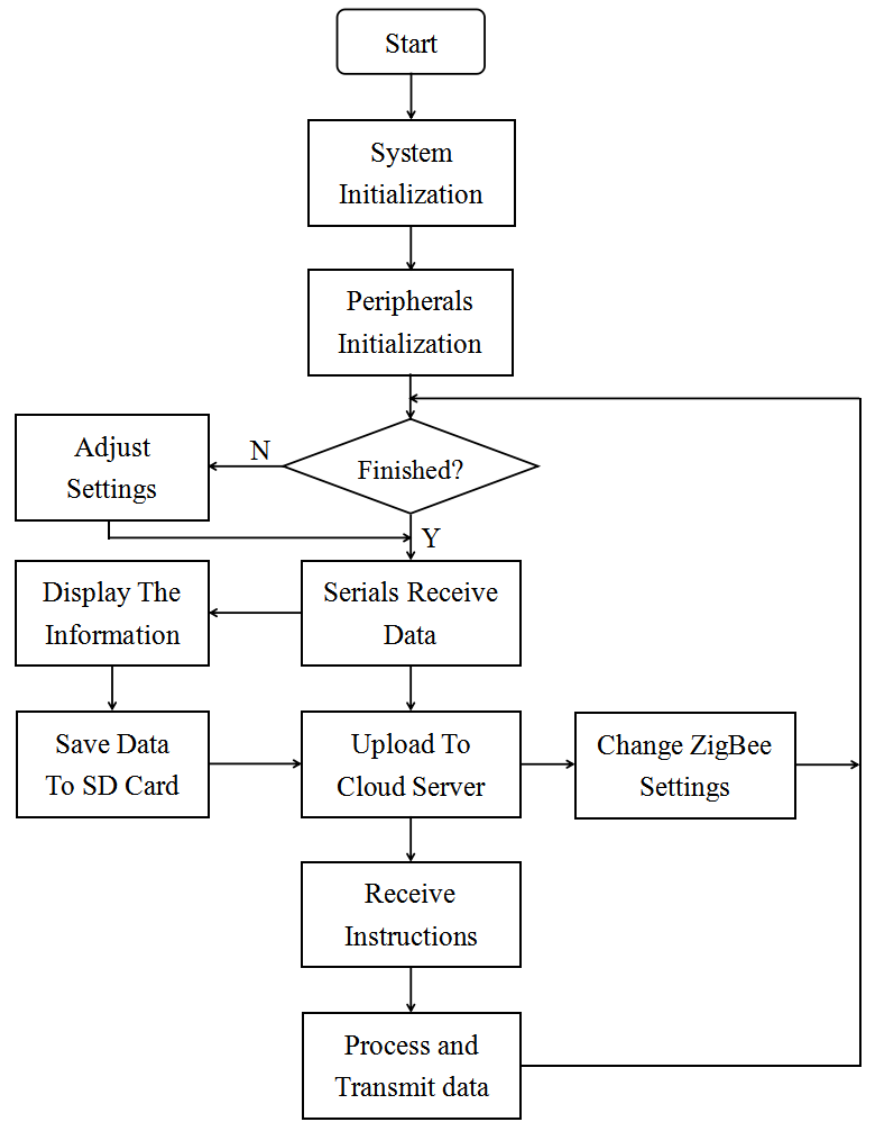

Fig. 5. The flowchart of manage center

\section{Simulation and Experimental Results}

\section{Simulation of system}

It is of great significance to study the average symbol rate of system with the increase of sensor nodes. The system chooses Network Simulator NS-2 as the network simulation platform, so as to verify the performance of system. The average data rate of nodes is shown as Fig. 6(a). Compared with traditional way, the rate of transmission is nearly constant, with the increase of nodes, the average rate increases, there are less data volume and data collision because of the data fusion algorithm. Therefore, the large-scale wireless sensor networks is effective for biogas digester monitoring system.

2. The feasibility test of system

To verify the feasibility of the monitoring system, we have developed wireless sensor network and GPRS intelligent gateway shown as Fig. 6(b). The wireless sensor network which applies CC2530 SOC as the core chip includes sensor nodes and routing nodes. The routing nodes is placed within the wireless signal coverage area of sensor nodes and GPRS intelligent gateway, so as to upload the data accurately. The GPRS intelligent gateway communicates with the nearby routing nodes, collects the environmental information and changes the settings of wireless sensor networks. Further more, the collected information can be acquired from LCDs of sensor nodes and gateway.

3. Upper supervision software

Upper supervision software has been widely used as a reliable tool to show environmental 
message precisely in monitoring system. As shown as Fig. 6(c), users are able to acquire the parameter configuration and real-time information such as temperature and humidity of sensor nodes. The software allows the supervision departments consult the historical trend curve of factors mentioned above and contribute resources to be shared with other users.

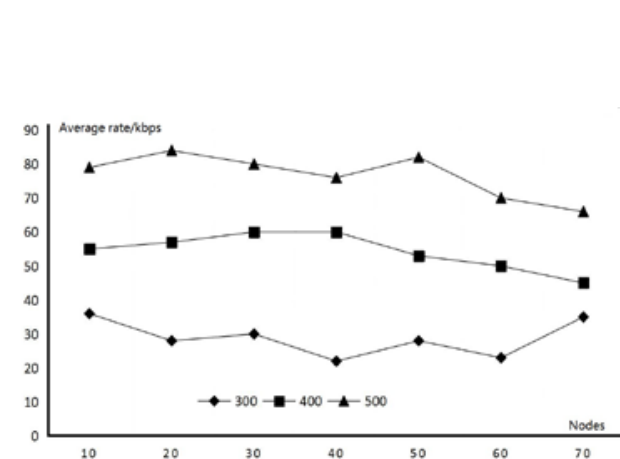

(a)

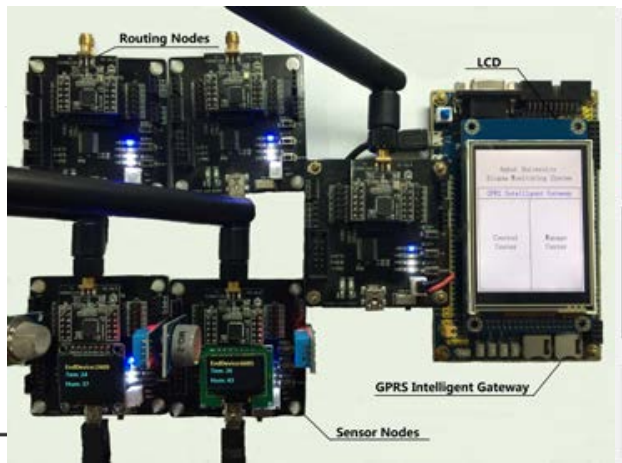

(b)

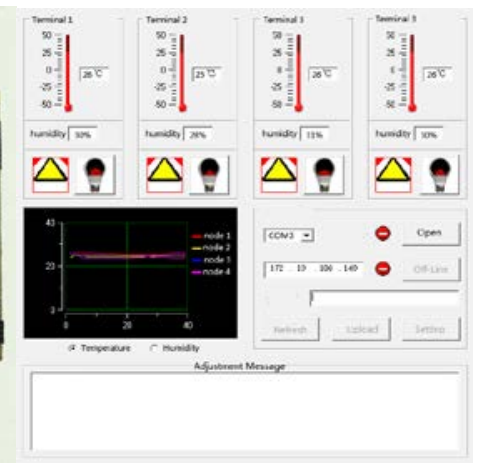

(c)

Fig. 6. Simulation and Experimental Results. (a) Average rate of transmission. (b) The devices of system. (c) The upper supervision software of system

\section{Conclusion}

Wireless sensor network has a great impact on our daily life for its features of low power cost, protocol flexibility, dynamic multi-routing and self-organization. This paper presents an intelligent biogas digester monitoring system based on wireless sensor network. Further more, a specific design method of multi-sensor data fusion and intelligent GPRS gateway with brilliant anti-interference is proposed.

The experimental results show that the hardware and software of monitoring system work properly, the system provides a specific way to accomplish intelligent and informational management of biogas digesters with low power consumption. Future work related on this subject includes the real-time communication and larger signal coverage of wireless sensor networks. Further on, the improvement of the acquisition speed and integration would also help to enhance the system performance.

\section{Acknowledgement}

In this paper, the research was sponsored by the Student's Platform for Innovation and Entrepreneurship Training Program(J1851169).

\section{Reference}

[1] Ding Tan, Wang Xuewen, Liu Changsheng, Xu Guimin, Wang Xuemei, Song Ling. Research of Biogas Power Generator System Based on Internet Technology. International Conference on Image Analysis \& Signal Processing, 2011:704-706.

[2] Wang Xiaohua, Li Jingfei. Influence of using household biogas digesters on household energy consumption in rural areas-a case study in Lianshui County in China. Renewable \& Sustainable Energy Reviews. 2005, 9(2):229-236.

[3] IF Akyildiz, W Su, Y Sankarasubramaniam, E Cayirci. Wireless sensor networks: a survey. Applied Mechanics \& Materials, 2013, 300-301(4):490-493.

[4] Zhao Han, Jie Wu, Jie Zhang, Liefeng Liu, Kaiyun Tian. A General Self-Organization Tree-Based Energy-Balance routing protocol for wireless sensor network. IEEE Transaction on Nuclear Science, 2012, 8537(11):1-6.

[5] Tuan Dinh Le, Dat Ho Tan. Design and Deploy a Wireless Sensor Network for Precision Agriculture. Conference on Information \& Computer Science, 2015. 
[6] Xiaonan Wang, Deguang Le, Hongbin Cheng, Conghua Xie. All-IP wireless sensor networks for real-time patient monitoring. Journal of Biomedical Informatics, 2014, 52:406-417.

[7] J Guevara, F Barrero, E Vargas, J Becerra, S Toral. Environmental wireless sensor network for road traffic applications. Let Intelligent Transport System, 2012, 6(2):177-186.

[8] Zhigao Liu, Chunwen Li, Danchen Wu, Wenhan Dai, Shaobo Geng, Qingqing Ding. A wireless sensor network based on personnel positioning scheme in coal mines with blind areas. Sensors, 2010, 10(11):9891-9918.

[9] Zhongying Wang, Tianyun Shi, Xiaojun Lv, Xinchun Jia, Wei Bai, Jun Hao. A tentative design of operating environmental monitoring and warning system of high-speed trains based on a Wireless Sensor Network. Control \& Decision Conference, 2015.

[10] Li Wenbin, Cui Dongxu, Zhang Junguo. Design and Implementation of Wireless Sensor Network Gateway Faced to Forest Information Monitor. Third International Conference on Intelligent System Design \& Engineering Applications, 2010, 1:524-526.

[11] Zhenxing Shi, Xiujuan Fan, Zhao Jing. Intelligent Environmental Monitoring System Design Based on CC2530. International Conference on Measuring Technology \& Mechatronics Automation, 2013:652-654.

[12] Gertjan Halkes, Koen Langendoen. Practical Considerations for Wireless Sensor Network Algorithms. Wireless Sensor Network, 2010, 2(6):441-446. 